\title{
Unscented Transform-based Dual Adaptive Control of Nonlinear MIMO Systems
}

\author{
Simon G. Fabri, Senior Member, IEEE and Marvin K. Bugeja, Member, IEEE
}

\begin{abstract}
The paper proposes a multilayer perceptron neural network controller for dual adaptive control of a class of stochastic MIMO nonlinear systems subject to functional uncertainty. The neural network parameters are adjusted in real-time using the Unscented Kalman filter algorithm and no pre-operational training phase is required. Dual adaptive control aims to strike a compromise between the two control characteristics of caution and probing, leading to an improved overall performance. The system is evaluated through numerical simulations and Monte Carlo analysis. The resulting performance of the dual adaptive controller is not only consistently superior to non-dual adaptive control schemes, but also surpasses the performance of similar controllers that are based on Extended Kalman filter estimators. This reflects the enhanced accuracy of the Unscented Kalman filter estimator, despite being a local estimation method. In addition, unlike use of other estimators, the proposed approach neither requires the computation of complex Jacobian matrices as part of the design, nor the evaluation of such matrices in real-time. This renders the proposed controller inherently amenable and practical for real-time implementation.
\end{abstract}

\section{INTRODUCTION}

Dual adaptive control is an adaptive control strategy based on stochastic control principles. It aims to generate a control signal which not only takes into consideration the degree of uncertainty present in the estimates of the plant's unknown features, but also probes the plant input so as to actively reduce this uncertainty. These two aims, referred to as caution and probing (hence dual control), aim to generate a control signal that is able to maintain an optimal tracking performance in the presence of uncertainty. This is obtained through optimization of a suitably-defined cost function [1], [2].

The ideal cost function which leads to an optimal dual controller entails the use of dynamic programming to solve the Bellman Equation, whose computation in this context inevitably results in a hugely impractical computational demand [3], [4]. It is thus common practice to design instead dual controllers that are based on a suboptimal cost function that leads to a tractable, albeit less optimal, solution but which somewhat retains the two desirable properties of dual control. Several dual suboptimal cost functions have been proposed in the literature [5]-[7].

In this paper we are concerned with dual adaptive control of a nonlinear class of multiple-input, multiple-output (MIMO) systems subject to functional uncertainty. A related but simpler problem was originally considered by Fabri

The authors are with the Department of Systems and Control Engineering, University of Malta, Msida MSD2080, Malta. (e-mail: simon.fabri@um.edu.mt, marvin.bugeja@um.edu.mt) and Kadirkamanathan [4], [8] for input-affine single-input, single-output (SISO) nonlinear systems. Both Gaussian basis function and multilayer perceptron (MLP) neural networks were considered as estimators for the unknown nonlinear functionals. Kalman filter and Extended Kalman filter (EKF) techniques were applied for neural network training and for generation of the control signal. The Innovations Dual Control (IDC) criterion, originally proposed by Milito et al. [6], was used as cost function. In [9] Bugeja and Fabri extended the SISO work in [4] and [8] to dual adaptive trajectory tracking control of a mobile robot, which is a specific example of MIMO systems. This was followed by Král and Šimandl's dual adaptive controller [10] for a more general MIMO class of nonlinear systems which utilizes Filatov and Unbehauen's Bicriterial cost function [5] instead of the IDC, and a Gaussian Sum Filter (GSF) for parameter estimation.

The main novel contribution reported in this paper is the extension of the adaptive controllers developed in [4], [8] and [9] to a generic class of MIMO nonlinear systems that are affine in the control input, utilizing MLP dual adaptive neuro-control laws. In addition, the use of Unscented Kalman filter (UKF) techniques [11] is proposed for a more accurate, efficient and real-time estimation of the MLP parameters and a less complex derivation of the dual adaptive control laws which does not require computation of the system's Jacobian matrices. This differs from [10] in two main aspects: (a) use of the UKF instead of the GSF as parameter estimator. As in all global estimation methods, the GSF requires higher computational demands than local estimation methods such as the UKF or EKF [12]; (b) avoidance of complex computation and on-line evaluation of the system's Jacobian matrices. The novel controller proposed in this paper inherently circumvents such issues, making the system more amenable and practical for real-time implementation.

The rest of the paper will present the problem formulation and mathematical preliminaries in Section II, followed by a description of the controller design in Section III. Section IV presents the performance results of the proposed controller together with a comparative analysis using Monte Carlo simulations, followed by conclusions in Section V.

\section{Problem Formulation}

We consider the following stochastic class of input-affine, nonlinear square MIMO systems in discrete-time $k$ having $s$ inputs and $s$ outputs:

$$
\boldsymbol{y}_{k}=\boldsymbol{f}\left(\boldsymbol{x}_{k-1}\right)+\boldsymbol{G}\left(\boldsymbol{x}_{k-1}\right) \boldsymbol{u}_{k-1}+\boldsymbol{\epsilon}_{k}
$$


where

- $\boldsymbol{y}_{k} \in \mathbb{R}^{s}$ is the output vector

- $\boldsymbol{u}_{k} \in \mathbb{R}^{s}$ is the control input vector

- $\boldsymbol{x}_{k-1}=\left[\begin{array}{ll}\boldsymbol{y}_{k-n}^{T} \ldots \boldsymbol{y}_{k-1}^{T} & \boldsymbol{u}_{k-1-p}^{T} \ldots \boldsymbol{u}_{k-2}^{T}\end{array}\right]^{T} \in$ $\mathbb{R}^{s(n+p)}$ is the state vector, where $0 \leq p \leq n$. The $\boldsymbol{u}$ terms vanish from the state vector if $p=0$

- vector $\boldsymbol{f}\left(\boldsymbol{x}_{k-1}\right): \mathbb{R}^{s(n+p)} \mapsto \mathbb{R}^{s}$ and matrix $\boldsymbol{G}\left(\boldsymbol{x}_{k-1}\right)$ : $\mathbb{R}^{s(n+p)} \mapsto \mathbb{R}^{s \times s}$ consist of unknown nonlinear functionals of the state vector

- $\epsilon_{k} \in \mathbb{R}^{s}$ is an additive noise signal.

The control objective is for the output vector to track a reference input vector $\boldsymbol{y}_{\boldsymbol{d}_{k}} \in \mathbb{R}^{s}$ under the following assumptions that are fairly standard in this type of work:

Assumption 1: The noise signal $\epsilon_{k}$ is statistically independent and has a white zero-mean Gaussian distribution with covariance $\boldsymbol{R}_{\boldsymbol{\epsilon}}$.

Assumption 2: The parameters $s, p, n$ and the noise covariance $\boldsymbol{R}_{\boldsymbol{\epsilon}}$ are known.

Assumption 3: The reference input $\boldsymbol{y}_{\boldsymbol{d}}$ is bounded and known at least one time step ahead i.e. $\boldsymbol{y}_{\boldsymbol{d}+1}$ is available at time $k$.

Assumption 4: $\boldsymbol{G}\left(\boldsymbol{x}_{k-1}\right)$ is bounded (has finite elements) and nonsingular. The latter constrains system (1) to be square.

Since the nonlinear functionals in $\boldsymbol{f}\left(\boldsymbol{x}_{k-1}\right)$ and $\boldsymbol{G}\left(\boldsymbol{x}_{k-1}\right)$ are unknown, the problem becomes one of adaptive control where two MLP neural networks are used to estimate and approximate these functionals within an arbitrarily large compact set $\chi$ in which the state vector is known to be contained. The estimates from the neural network will be utilized by the dual adaptive control law. The structure of the neural networks, both with one hidden layer, is depicted in Figure 1. Their outputs are given by (2) and (3) for the networks approximating $\boldsymbol{f}\left(\boldsymbol{x}_{k-1}\right)$ and $\boldsymbol{G}\left(\boldsymbol{x}_{k-1}\right)$ respectively, where $\tilde{\boldsymbol{f}} \in \mathbb{R}^{s}$ and $\tilde{\boldsymbol{G}} \in \mathbb{R}^{s \times s}$.

$$
\tilde{\boldsymbol{f}}_{k-1}=\left[\begin{array}{c}
\boldsymbol{\phi}_{\boldsymbol{f}}^{T}\left(\boldsymbol{x}_{k-1}, \hat{\boldsymbol{a}}_{k}\right) \hat{\boldsymbol{w}}_{1 k} \\
\boldsymbol{\phi}_{\boldsymbol{f}}^{T}\left(\boldsymbol{x}_{k-1}, \hat{\boldsymbol{a}}_{k}\right) \hat{\boldsymbol{w}}_{2 k} \\
\vdots \\
\boldsymbol{\phi}_{\boldsymbol{f}}^{T}\left(\boldsymbol{x}_{k-1}, \hat{\boldsymbol{a}}_{k}\right) \hat{\boldsymbol{w}}_{s k}
\end{array}\right]
$$

The terms in these neural network equations are defined as:

Definition 1: $\hat{\boldsymbol{w}}_{i k}$ represents the synaptic weight estimate vector of the connection between the neuron hidden layer and the $i^{\text {th }}$ element of the first neural network output $\tilde{\boldsymbol{f}}_{k-1}$. Similarly, $\hat{\boldsymbol{v}}_{i, j k}$ represents the synaptic weight estimate vector of the connection between the neuron hidden layer and the $(i, j)^{\text {th }}$ element of the second neural network output $\tilde{G}_{k-1}$. The ${ }^{\wedge}$ notation is used to indicate that the operand is undergoing estimation.

Definition 2: $\phi_{f}$ and $\phi_{G}$ are vectors of sigmoidal activation functions whose $i^{\text {th }}$ element is respectively given by

$$
\begin{aligned}
\phi_{f_{(i)}} & =\left(1+\exp \left(-\hat{\boldsymbol{s}}_{i}{ }^{T} \check{\boldsymbol{x}}\right)\right)^{-1}, \quad i=1, \ldots, L_{f} \\
\phi_{G(i)} & =\left(1+\exp \left(-\hat{\boldsymbol{t}}_{i}^{T} \check{\boldsymbol{x}}\right)\right)^{-1}, \quad i=1, \ldots, L_{G}
\end{aligned}
$$

where $\check{\boldsymbol{x}}=\left[\begin{array}{ll}\boldsymbol{x}^{T} & 1\end{array}\right]^{T}$ (of length $L_{a}$ ) denotes the system state vector augmented by an additional constant input serving as a bias term. $\hat{\boldsymbol{s}}_{i}$ and $\hat{\boldsymbol{t}}_{i}$ (both having the same length as $\check{\boldsymbol{x}}$ ) serve to shape the hidden layer units and denote the $i^{\text {th }}$ vectors in the group vectors $\hat{\boldsymbol{a}}, \hat{\boldsymbol{b}}$ respectively i.e. $\hat{\boldsymbol{a}}=\left[\begin{array}{lll}\hat{\boldsymbol{s}}_{1}^{T} & \ldots & \hat{\boldsymbol{s}}_{L_{f}}^{T}\end{array}\right]^{T}$ and $\hat{\boldsymbol{b}}=\left[\begin{array}{lll}\hat{\boldsymbol{t}}_{1}^{T} & \cdots & \hat{\boldsymbol{t}}_{L_{G}}^{T}\end{array}\right]^{T}$ representing the hidden neuron parameters. $L_{f}$ and $L_{G}$ denote the number of sigmoidal activation functions and the time index, which is the same on both sides, has been dropped for clarity.

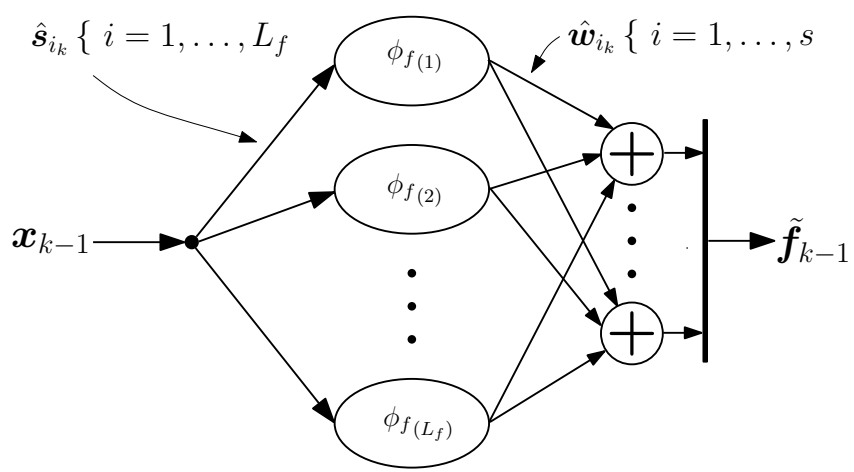

(a) neural network for $\boldsymbol{f}\left(\boldsymbol{x}_{k-1}\right)$

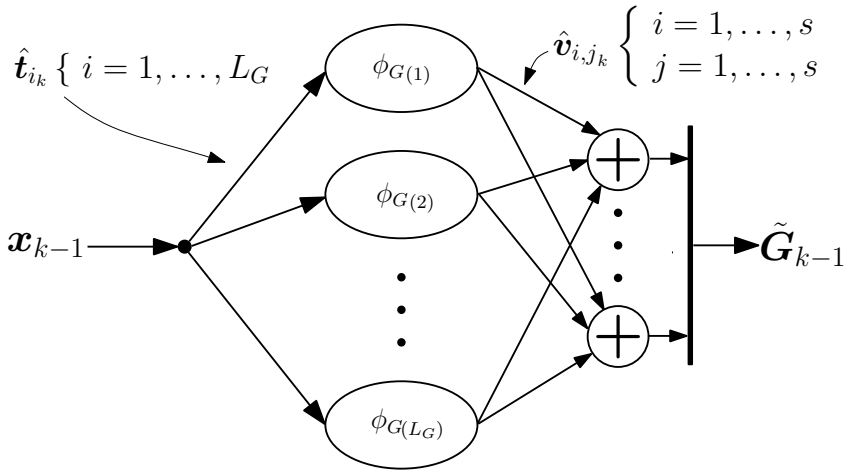

(b) neural network for $\boldsymbol{G}\left(\boldsymbol{x}_{k-1}\right)$

Fig. 1: Sigmoidal multilayer perceptron neural networks.

$$
\tilde{\boldsymbol{G}}_{k-1}=\left[\begin{array}{cccc}
\phi_{G}^{T}\left(\boldsymbol{x}_{k-1}, \hat{\boldsymbol{b}}_{k}\right) \hat{\boldsymbol{v}}_{1,1 k} & \phi_{G}^{T}\left(\boldsymbol{x}_{k-1}, \hat{\boldsymbol{b}}_{k}\right) \hat{\boldsymbol{v}}_{1,2 k} & \cdots & \phi_{G}^{T}\left(\boldsymbol{x}_{k-1}, \hat{\boldsymbol{b}}_{k}\right) \hat{\boldsymbol{v}}_{1, s k} \\
\boldsymbol{\phi}_{G}^{T}\left(\boldsymbol{x}_{k-1}, \hat{\boldsymbol{b}}_{k}\right) \hat{\boldsymbol{v}}_{2,1 k} & \phi_{\boldsymbol{G}}^{T}\left(\boldsymbol{x}_{k-1}, \hat{\boldsymbol{b}}_{k}\right) \hat{\boldsymbol{v}}_{2,2 k} & \cdots & \phi_{G}^{T}\left(\boldsymbol{x}_{k-1}, \hat{\boldsymbol{b}}_{k}\right) \hat{\boldsymbol{v}}_{2, s k} \\
\vdots & \vdots & \ddots & \vdots \\
\boldsymbol{\phi}_{G}^{T}\left(\boldsymbol{x}_{k-1}, \hat{\boldsymbol{b}}_{k}\right) \hat{\boldsymbol{v}}_{s, 1 k} & \phi_{G}^{T}\left(\boldsymbol{x}_{k-1}, \hat{\boldsymbol{b}}_{k}\right) \hat{\boldsymbol{v}}_{s, 2 k} & \cdots & \boldsymbol{\phi}_{G}^{T}\left(\boldsymbol{x}_{k-1}, \hat{\boldsymbol{b}}_{k}\right) \hat{\boldsymbol{v}}_{s, s k}
\end{array}\right]
$$


Let all the neural network synaptic weights and hidden neuron parameters be grouped into a single weights vector $\hat{\boldsymbol{z}}_{k}$ defined as follows:

$$
\begin{aligned}
& \text { Definition 3: } \hat{\boldsymbol{z}}_{k} \triangleq\left[\begin{array}{ll}
\hat{\boldsymbol{q}}_{k}^{T} & \hat{\boldsymbol{r}}_{k}^{T}
\end{array}\right]^{T} \text {, where } \\
& \hat{\boldsymbol{q}}_{k}=\left[\begin{array}{lll}
\hat{\boldsymbol{w}}_{1 k}^{T} \ldots \hat{\boldsymbol{w}}_{s k}^{T} & \hat{\boldsymbol{a}}_{k}^{T}
\end{array}\right]^{T}
\end{aligned}
$$

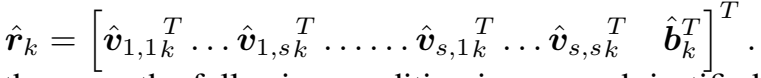

Furthermore the following condition is assumed, justified by the neural network Universal Approximation Property [13]:

Assumption 5: Inside the compact set $\chi$, there exists an optimal value for the weights vector $\hat{\boldsymbol{z}}_{k}$, denoted by $\boldsymbol{z}_{k}^{*}$, such that the neural network approximation errors are negligibly small.

This optimal value of $\hat{\boldsymbol{z}}_{k}$ is unknown and thus requires estimation. Utilizing Definitions 1 to 3, Equations (2), (3) and Assumption 5, then within $\chi$ the MIMO system (1) can be represented in the following state space form:

$$
\begin{aligned}
\boldsymbol{z}_{k+1}^{*} & =\boldsymbol{z}_{k}^{*}+\boldsymbol{\rho}_{k} \\
\boldsymbol{y}_{k} & =\boldsymbol{h}\left(\boldsymbol{x}_{k-1}, \boldsymbol{u}_{k-1}, \boldsymbol{z}_{k}^{*}\right)+\boldsymbol{\epsilon}_{k},
\end{aligned}
$$

where the vector-valued function $\boldsymbol{h}\left(\boldsymbol{x}_{k-1}, \boldsymbol{u}_{k-1}, \boldsymbol{z}_{k}^{*}\right)$ is nonlinear in $z_{k}^{*}$ and is given by

$$
\boldsymbol{h}\left(\boldsymbol{x}_{k-1}, \boldsymbol{u}_{k-1}, \boldsymbol{z}_{k}^{*}\right)=\tilde{\boldsymbol{f}}\left(\boldsymbol{x}_{k-1}, \boldsymbol{q}_{k}^{*}\right)+\tilde{\boldsymbol{G}}\left(\boldsymbol{x}_{k-1}, \boldsymbol{r}_{k}^{*}\right) \boldsymbol{u}_{k-1} .
$$

Furthermore, an additive white noise term $\boldsymbol{\rho}_{k}$, taken to be Gaussian distributed with zero mean and covariance $\boldsymbol{Q}_{\boldsymbol{\rho}}$, is introduced in (4) so as to model any small errors resulting from Assumption 5. This so-called process noise term has the additional benefit of aiding parameter convergence during the estimation process.

\section{Controller Design}

Dual control design is based upon a stochastic approach. Thus the unknown optimal weights vector $z_{k}^{*}$ is modelled as a random variable with initial condition $\boldsymbol{z}_{0}^{*}$ taken to be Gaussian distributed with mean $\hat{\boldsymbol{z}}_{0}$ and covariance $\boldsymbol{P}_{0}$. It is additionally assumed that the output noise term $\epsilon_{k}$, the process noise $\boldsymbol{\rho}_{k}$ and the initial condition $\hat{\boldsymbol{z}}_{0}$ are mutually independent for all $k$. In effect, under these considerations and Assumption 1, Equation (4) represents a nonlinear stochastic state space model subject to additive Gaussian noise terms.

Since the output equation in (4) is nonlinear in the optimal weights vector term, the distribution of the random variables will not remain Gaussian. Hence nonlinear estimation techniques must be applied in order to compute the probability distribution of these variables and its propagation through time. In this work we opt to use the UKF as a nonlinear estimator [11], [14]. The UKF uses a Gaussian density function to approximate the non-Gaussian distribution but it is specified using a set of sample points, called sigma points, which when propagated through the nonlinear system dynamics, capture the posteriors more accurately than the EKF, up to second order. The GSF, which approximates non-Gaussian distributions through a summation of several
Gaussian components, can achieve better accuracy in general. However, being based on multiple components whose quantity may also increase at each time step unless pruning is applied [15], the GSF is much more computationally demanding than the UKF.

The dual adaptive control law developed in this paper is based upon the explicit-type IDC cost function (6) adopted from [4], [6], [8] and redesigned to fit the nonlinear MIMO system under consideration, namely:

$$
\begin{array}{r}
J_{i n n}=E\left\{\left(\boldsymbol{y}_{k+1}-\boldsymbol{y}_{\boldsymbol{d}_{k+1}}\right)^{T} \boldsymbol{Q}_{\mathbf{1}}\left(\boldsymbol{y}_{k+1}-\boldsymbol{y}_{\boldsymbol{d}_{k+1}}\right)+\right. \\
\left.\boldsymbol{u}_{k}^{T} \boldsymbol{Q}_{\mathbf{2}} \boldsymbol{u}_{k}+\boldsymbol{i}_{k+1}^{T} \boldsymbol{Q}_{\mathbf{3}} \boldsymbol{i}_{k+1} \mid I^{k}\right\}
\end{array}
$$

where $E\left\{\cdot \mid I^{k}\right\}$ denotes mathematical expectation conditioned upon the information state $I^{k} \triangleq$ $\left\{\boldsymbol{y}_{k} \cdots \boldsymbol{y}_{0} \boldsymbol{u}_{k} \cdots \boldsymbol{u}_{0}\right\}$. This cost function explicitly includes the estimator's innovations vector $\boldsymbol{i}_{k+1}$ by means of which we can influence the extent to which the innovations remain high, thereby driving the estimator by richer information which could improve and accelerate the estimation process. In this manner a controlled level of probing is introduced. The weighting matrices $\boldsymbol{Q}_{\mathbf{1}}$ and $\boldsymbol{Q}_{\mathbf{2}}$ are $(s \times s)$ diagonal matrices with real positive elements. Hence $\boldsymbol{Q}_{\mathbf{1}}$ and $\boldsymbol{Q}_{\mathbf{2}}$ are positive-definite. $\boldsymbol{Q}_{\mathbf{3}}$ is also an $(s \times s)$ diagonal matrix, and each of its diagonal elements is less than or equal to zero, and greater than or equal to the corresponding element in $-Q_{1}$, i.e. $-Q_{1} \leq Q_{3} \leq 0$ (element-wise).

Weighting matrix $Q_{1}$ introduces a penalty on tracking errors, $\boldsymbol{Q}_{\mathbf{2}}$ penalizes large control inputs, and $\boldsymbol{Q}_{\mathbf{3}}$ is used to adjust the weight of the innovations vector within the cost function. The latter is what induces the dual-like effect characterizing the proposed stochastic control law.

\section{A. The UKF estimator}

The estimator recursively generates a predictive estimate $\hat{\boldsymbol{z}}_{k+1 \mid k}$ of the optimal weights vector $\boldsymbol{z}_{k+1}^{*}$ at every time instant $k$ for use by the dual adaptive control law. The prediction is required because of the relative degree of one appearing in the system equation. The mean of the nonGaussian conditional distribution of $\boldsymbol{z}_{k+1}^{*}$ given $I^{k}$ represents a good choice for such an estimate. The UKF approximates this distribution by a Gaussian density function. We thus take the mean of the latter to approximate the required predictive estimate $\hat{z}_{k+1 \mid k}$. The covariance of the Gaussian distribution from the UKF, $\boldsymbol{P}_{k+1 \mid k}$ can be viewed as a measure of this estimate's uncertainty. These two statistics are computed at each time instant $k$ according to the following UKF procedure which is largely the same as that in [14] but modified slightly so as to generate predictive instead of filtered updates. At any time step $k$, given the previous prediction $\left(\hat{z}_{k \mid k-1}, \boldsymbol{P}_{k \mid k-1}\right)$, calculate $\left(\hat{\boldsymbol{z}}_{k+1 \mid k}, \boldsymbol{P}_{k+1 \mid k}\right)$ by first forming the matrix $\mathcal{Z}_{k \mid k-1}$ of $2 N+1$ sigma point 
vectors, where $N$ denotes the length of $\hat{\boldsymbol{z}}_{k}$, as follows:

$$
\begin{aligned}
& \mathcal{Z}_{(:, 1)_{k}}=\hat{\boldsymbol{z}}_{k \mid k-1} \\
& \mathcal{Z}_{(:, i)_{k}}=\hat{\boldsymbol{z}}_{k \mid k-1}+\gamma{\sqrt{\boldsymbol{P}_{k \mid k-1}}}_{(:, i)}, i=2, \ldots, N+1 \\
& \mathcal{Z}_{(:, i)_{k}}=\hat{\boldsymbol{z}}_{k \mid k-1}-\gamma{\sqrt{\boldsymbol{P}_{k \mid k-1}}}_{(:, i)}, i=N+2, \ldots, 2 N+1
\end{aligned}
$$

where the notation $\boldsymbol{A}_{(:, i)}$ denotes the $i^{t h}$ column vector of any given matrix $\boldsymbol{A}, \sqrt{\boldsymbol{P}_{k \mid k-1}}$ denotes a matrix square rootlike operation such as lower-triangular Cholesky factorization, and $\gamma=\sqrt{N+\lambda}$ where $\lambda$ is a scaling parameter given by $\lambda=\alpha^{2}(N+\kappa)-N$. The constant $\alpha$ determines the spread of the sigma points around $\hat{\boldsymbol{z}}_{k}$ and is usually set to a small positive value, and the constant $\kappa$ is a secondary scaling parameter typically set to $3-N$ [14].

The sigma point vectors are then propagated through (4) and (5), the system dynamic equations, as follows:

$$
\begin{aligned}
\mathcal{F}_{(:, i)_{k-1}} & =\tilde{\boldsymbol{f}}\left(\boldsymbol{x}_{k-1}, \boldsymbol{\mathcal { Z }}_{\left.\boldsymbol{q}_{(:, i)_{k}}\right)}\right) \\
\mathcal{G}_{(:,:, i)_{k-1}} & =\tilde{\boldsymbol{G}}\left(\boldsymbol{x}_{k-1}, \mathcal{Z}_{\boldsymbol{r}(:, i)_{k}}\right) \\
\mathcal{Y}_{(:, i)_{k}} & =\mathcal{F}_{(:, i)_{k-1}}+\mathcal{G}_{(:,:, i)_{k-1}} \boldsymbol{u}_{k-1}
\end{aligned}
$$

where $i=1, \ldots, 2 N+1$, the notation $\boldsymbol{A}_{(:,:, i)}$ denotes the $i^{\text {th }}$ matrix from a group of matrices $\boldsymbol{A}$, and $\mathcal{Z}_{\boldsymbol{q}_{k}}$, $\mathcal{Z}_{\boldsymbol{r} k}$ are submatrices of size $\left(L_{f}\left(s+L_{a}\right) \times 2 N+1\right)$ and $\left(L_{G}\left(s^{2}+L_{a}\right) \times 2 N+1\right)$ respectively, from the matrix of sigma vectors $\mathcal{Z}_{k}$ after its columns are repartitioned according to Definition 3 i.e. $\mathcal{Z}_{k}=\left[\begin{array}{ll}\mathcal{Z}_{\boldsymbol{q}_{k}}^{T} & \mathcal{Z}_{\boldsymbol{r} k}^{T}\end{array}\right]^{T}$.

These are then used to approximate the mean and covariance of the estimated output vector $\hat{\boldsymbol{y}}_{k}$ using a weighted sample mean and covariance from the propagated sigma points $\mathcal{Y}_{k}$ :

$$
\begin{aligned}
\hat{\boldsymbol{y}}_{k \mid k-1}= & \sum_{i=1}^{2 N+1} W_{m(i)} \mathcal{Y}_{(:, i)_{k}} \\
\boldsymbol{P}_{\boldsymbol{y} \boldsymbol{y}_{k \mid k-1}=} & \sum_{i=1}^{2 N+1} W_{c(i)}\left(\mathcal{Y}_{(:, i)_{k}}-\hat{\boldsymbol{y}}_{k}\right)\left(\mathcal{Y}_{(:, i)_{k}}-\hat{\boldsymbol{y}}_{k \mid k-1}\right)^{T} \\
& +\boldsymbol{R}_{\boldsymbol{\epsilon}} \\
& 2{ }_{\boldsymbol{z y}_{k \mid k-1}}=\sum_{i=1}^{N+1} W_{c(i)}\left(\boldsymbol{\mathcal { Z }}_{(:, i)_{k}}-\hat{\boldsymbol{z}}_{k}\right)\left(\mathcal{Y}_{(:, i)_{k}}-\hat{\boldsymbol{y}}_{k \mid k-1}\right)^{T}
\end{aligned}
$$

where the above so-called Unscented Transform weights are:

$$
\begin{aligned}
W_{m(1)} & =\frac{\lambda}{N+\lambda} \\
W_{c(1)} & =\frac{\lambda}{N+\lambda}+1-\alpha^{2}+\beta \\
W_{m(i)} & =W_{c(i)}=\frac{1}{2(N+\lambda)}, \quad i=2, \ldots, 2 N+1
\end{aligned}
$$

The constant parameter $\beta$ is used to incorporate prior knowledge of the estimate's distribution (for a Gaussian, $\beta=2$ [14]). The predictive estimates $\left(\hat{\boldsymbol{z}}_{k+1 \mid k}, \boldsymbol{P}_{k+1 \mid k}\right)$ are then given by:

$$
\begin{aligned}
\hat{\boldsymbol{z}}_{k+1 \mid k} & =\hat{\boldsymbol{z}}_{k \mid k-1}+\boldsymbol{K}_{k} \boldsymbol{i}_{k} \\
\boldsymbol{P}_{k+1 \mid k} & =\boldsymbol{P}_{k \mid k-1}-\boldsymbol{K}_{k} \boldsymbol{P}_{\boldsymbol{y} \boldsymbol{y}_{k \mid k-1}} \boldsymbol{K}_{k}^{T}+\boldsymbol{Q}_{\boldsymbol{\rho}}
\end{aligned}
$$

where $\boldsymbol{K}_{k}=\boldsymbol{P}_{\boldsymbol{z y}} \boldsymbol{P}_{k \mid k-1} \boldsymbol{P}_{\boldsymbol{y} \boldsymbol{y}_{k \mid k-1}}^{-1}$ represents the Kalman Gain and $\boldsymbol{i}_{k}=\boldsymbol{y}_{k}-\hat{\boldsymbol{y}}_{k \mid k-1}$ is the innovations vector.

\section{B. The control law}

The minimization of cost function (6) requires estimation of the probability density function of $\boldsymbol{y}_{k+1} \mid I^{k}$. Following the UKF methodology, this is approximated by a Gaussian of mean $\hat{\boldsymbol{y}}_{k+1 \mid k}$ and covariance $\boldsymbol{P}_{\boldsymbol{y} \boldsymbol{y}_{k+1 \mid k}}$ given by:

$$
\hat{\boldsymbol{y}}_{k+1 \mid k}=\hat{\boldsymbol{f}}_{k}+\hat{\boldsymbol{G}}_{k} \boldsymbol{u}_{k}
$$

where

$$
\hat{\boldsymbol{f}}_{k}=\sum_{i=1}^{2 N+1} W_{m(i)} \mathcal{F}_{(:, i)_{k}} \text { and } \hat{\boldsymbol{G}}_{k}=\sum_{i=1}^{2 N+1} W_{m(i)} \mathcal{G}_{(:,:, i)_{k}}
$$

and covariance:

$$
\begin{aligned}
& \boldsymbol{P}_{\boldsymbol{y}_{k+1 \mid k}}=\boldsymbol{R}_{\boldsymbol{\epsilon}}+ \\
& \sum_{i=1}^{2 N+1} W_{c(i)}\left(\mathcal{D}_{\boldsymbol{f}_{(:, i)}}+\mathcal{D}_{\boldsymbol{G}(:, i, i)} \boldsymbol{u}_{k}\right)\left(\mathcal{D}_{\boldsymbol{f}_{(:, i)}}+\mathcal{D}_{\boldsymbol{G}(:,: i)} \boldsymbol{u}_{k}\right)^{T}
\end{aligned}
$$

where

$$
\mathcal{D}_{\boldsymbol{f}_{(:, i)}}=\mathcal{F}_{(:, i)_{k}}-\hat{\boldsymbol{f}}_{k} \text { and } \mathcal{D}_{\boldsymbol{G}(:,:, i)}=\mathcal{G}_{(:,:, i)_{k}}-\hat{\boldsymbol{G}}_{k}
$$

These equations follow directly by applying the Unscented Transform and its related Gaussian approximations to calculate the statistics of $\boldsymbol{z}_{k+1}^{*}$ after undergoing the nonlinear transformation $\boldsymbol{h}\left(\boldsymbol{x}_{k}, \boldsymbol{u}_{k}, \boldsymbol{z}_{k+1}^{*}\right)$.

Theorem 1: The control law minimizing cost function $J_{i n n}$ of (6) subject to the nonlinear dynamic system (1) and all previous definitions, assumptions and preliminary results derived in the formulation of the UT-based scheme is given by

$$
\begin{aligned}
\boldsymbol{u}_{k}= & \left(\hat{\boldsymbol{G}}_{k}^{T} \boldsymbol{Q}_{\mathbf{1}} \hat{\boldsymbol{G}}_{k}+\boldsymbol{Q}_{\mathbf{2}}+\boldsymbol{N}_{\boldsymbol{G} \boldsymbol{G} k}\right)^{-1} \\
& \times\left(\hat{\boldsymbol{G}}_{k}^{T} \boldsymbol{Q}_{\mathbf{1}}\left(\boldsymbol{y}_{\boldsymbol{d}+1}-\hat{\boldsymbol{f}}_{k}\right)-\boldsymbol{\kappa}_{\boldsymbol{G} \boldsymbol{f}_{k}}\right)
\end{aligned}
$$

where $\hat{\boldsymbol{f}}_{k}$ and $\hat{\boldsymbol{G}}_{k}$ are computed via (8) and the auxiliary terms $\boldsymbol{\kappa}_{\boldsymbol{G} \boldsymbol{f}_{k}}$ and $\boldsymbol{N}_{\boldsymbol{G G k}}$ are given by

$$
\begin{aligned}
\boldsymbol{\kappa}_{\boldsymbol{G} \boldsymbol{f}_{k}} & =\sum_{i=1}^{2 N+1} W_{c(i)} \mathcal{D}_{\boldsymbol{G}_{(:,:, i)}{ }^{T} \boldsymbol{Q}_{\mathbf{4}} \mathcal{D}_{\boldsymbol{f}_{(:, i)}}} \\
\boldsymbol{N}_{\boldsymbol{G G} k} & =\sum_{i=1}^{2 N+1} W_{c(i)} \mathcal{D}_{\boldsymbol{G}(:,:, i)}^{T} \boldsymbol{Q}_{\mathbf{4}} \mathcal{D}_{\boldsymbol{G}(:,:, i)}
\end{aligned}
$$

with $\mathcal{D}_{\boldsymbol{G}(:, i, i)}$ and $\mathcal{D}_{\boldsymbol{f}_{(:, i)}}$ computed as in (10) and matrix $Q_{4} \triangleq Q_{1}+Q_{3}$.

Proof: Given the approximate Gaussian distribution of $\boldsymbol{y}_{k+1} \mid I^{k}$ specified by the mean and covariance (7) and (9), and standard results from multivariate probability theory, it follows that cost function (6) can be rewritten as:

$$
\begin{aligned}
J_{i n n}= & \left(\hat{\boldsymbol{y}}_{k+1 \mid k}-\boldsymbol{y}_{\boldsymbol{d} k+1}\right)^{T} \boldsymbol{Q}_{\mathbf{1}}\left(\hat{\boldsymbol{y}}_{k+1 \mid k}-\boldsymbol{y}_{\boldsymbol{d} k+1}\right) \\
& +\operatorname{tr}\left(\boldsymbol{Q}_{\mathbf{4}} \boldsymbol{P}_{\boldsymbol{y} \boldsymbol{y}_{k+1 \mid k}}\right)+\boldsymbol{u}_{k}^{T} \boldsymbol{Q}_{\mathbf{2}} \boldsymbol{u}_{k} .
\end{aligned}
$$


By substituting for $\hat{\boldsymbol{y}}_{k+1 \mid k}$ and $\boldsymbol{P}_{\boldsymbol{y} \boldsymbol{y}_{k+1 \mid k}}$ in (14), $J_{i n n}$ can be factorized completely in terms of $\boldsymbol{u}_{k}$. The resulting quadratic function is differentiated with respect to $\boldsymbol{u}_{k}$ and equated to zero in order to determine the stationary point. This leads to the dual control law (11). Moreover, the Hessian matrix obtained by the second derivative of $J_{i n n}$ with respect to $\boldsymbol{u}_{k}$ yields

$$
\frac{\partial}{\partial \boldsymbol{u}_{k}}\left(\frac{\partial J_{i n n}}{\partial \boldsymbol{u}_{k}}\right)=2\left(\hat{\boldsymbol{G}}_{k}^{T} \boldsymbol{Q}_{\mathbf{1}} \hat{\boldsymbol{G}}_{k}+\boldsymbol{Q}_{\mathbf{2}}+\boldsymbol{N}_{\boldsymbol{G} \boldsymbol{G} k}\right) .
$$

Since $Q_{\mathbf{1}}$ and $\boldsymbol{Q}_{\mathbf{2}}$ are positive-definite and $\boldsymbol{Q}_{\mathbf{4}}$ is positivesemidefinite, it follows that the above Hessian matrix is positive-definite subject to the non-stringent condition that $W_{c(i)}>0 \forall i$, in order to ensure that $\boldsymbol{N}_{\boldsymbol{G G}}$ in (13) remains positive-semidefinite. Hence, the dual adaptive control law in Theorem 1 minimizes uniquely the selected performance index $J_{i n n}(6)$ in the context of this scheme, and the inverse term in the control law exists without exceptions.

It is worth noting the effect of the innovations weighting matrix $\boldsymbol{Q}_{\mathbf{3}}$, appearing in cost function (6), on matrix $\boldsymbol{Q}_{4}$ and consequently the control law (11). At one extreme, with $Q_{3}=-Q_{1}$, matrix $Q_{4}$ becomes zero which in turn makes the terms $\boldsymbol{\kappa}_{\boldsymbol{G} \boldsymbol{f}_{k}}, \boldsymbol{N}_{\boldsymbol{G G} \boldsymbol{G}_{k}}$ vanish in (11). The controller thus ignores completely the estimates' uncertainty which leads to a Heuristic Certainty Equivalent (HCE) control law. At the other extreme, with $Q_{\mathbf{3}}=\mathbf{0}$, maximum attention is given to the uncertainty terms in (11), which leads to cautious control. Both these extremes are inferior to dual control in terms of performance [4]. HCE control leads to large tracking errors and excessive control actions when the estimates' uncertainty is relatively high, whereas cautious control is notorious for a sluggish response and control turnoff. For intermediate settings of $\boldsymbol{Q}_{\mathbf{3}}$, the controller strikes a compromise and operates in dual adaptive mode by obtaining a balance between these two extremes of excessive caution or aggressive control action, leading to a greatly improved performance.

\section{RESULTS}

The dual adaptive control scheme proposed in this paper is tested and validated using numerical simulations of a plant that satisfies the stochastic MIMO-square, input-affine model defined in (1) with the unknown nonlinear functionals in $\boldsymbol{f}\left(\boldsymbol{x}_{k-1}\right)$ and $\boldsymbol{G}\left(\boldsymbol{x}_{k-1}\right)$ given by:

$$
\begin{aligned}
\boldsymbol{f}\left(\boldsymbol{x}_{k-1}\right) & =\left[\begin{array}{c}
\sin x_{(1)} \cos x_{(2)} \\
\cos \left(x_{(1)}+x_{(2)}\right)
\end{array}\right] \\
\boldsymbol{G}\left(\boldsymbol{x}_{k-1}\right) & =\left[\begin{array}{cc}
2+\sin x_{(1)} & \cos x_{(2)} \\
-\cos \left(2 x_{(1)}+x_{(2)}\right) & 1+\sin x_{(2)}^{2}
\end{array}\right]
\end{aligned}
$$

where $s=2, n=1, p=0$ and $x_{(1)}$ and $x_{(2)}$ are the elements of state vector $\boldsymbol{x}$ which in this example is equal to $\boldsymbol{y} . \boldsymbol{G}\left(\boldsymbol{x}_{k-1}\right)$ satisfies Assumption 4. The noise covariance $\boldsymbol{R}_{\boldsymbol{\epsilon}}=0.0015 \mathbf{I}_{\mathbf{2}}$, where $\mathbf{I}_{\boldsymbol{i}}$ denotes an $(i \times i)$ identity matrix. The reference input vector $\boldsymbol{y}_{\boldsymbol{d}_{k}} \in \mathbb{R}^{2}$ satisfies Assumption 3 and consists of two terms: the first term $y_{d(1)}$ is generated by sampling a unit amplitude $5 \mathrm{~Hz}$ square wave filtered through a network of transfer function $1 /(0.5 s+1)$, and the second term $y_{d(2)}=-y_{d(1)}$. The two MLP neural networks used to estimate $\boldsymbol{f}\left(\boldsymbol{x}_{k-1}\right)$ and $\boldsymbol{G}\left(\boldsymbol{x}_{k-1}\right)$ are structured with $L_{f}=$ 6 and $L_{G}=5$ neurons, resulting in a total of $N=65$ parameters for estimation. The process noise covariance $\boldsymbol{Q}_{\boldsymbol{\rho}}$ is set to $10^{-8} \mathbf{I}_{N}$ and the initial condition and covariance of $z_{0}^{*}$ are set with the elements of $\hat{z}_{0}$ generated at random and $\boldsymbol{P}_{0}=1.0 \mathbf{I}_{\boldsymbol{N}}$. The UKF parameters are set to $\alpha=10^{-3}, \kappa=$ $3-N$ and $\beta=2$.

Figure 2 shows the typical output response of a single trial when the controller is tuned to give $\operatorname{HCE}\left(Q_{3}=-Q_{1}\right)$, cautious $\left(Q_{3}=0\right)$ and dual adaptive $\left(Q_{3}=-0.7 Q_{1}\right)$ control modes respectively. Plots (a) and (b) show that in HCE mode, the controller leads to an inadequate transient response characterized by relatively large output overshoots. Note that, for visual clarity, the $y$-axis of these two specific plots is truncated and peak overshoot in excess of 2 units was actually obtained. This is a direct result of the aggressive and incautious nature of the HCE approach which completely ignores the high uncertainty in the initial parameter estimates and uses them as if they were perfectly true. In cautious mode (plots (c) and (d)) and dual mode (plots ((e) and (f)), both controllers exhibit significantly lower transient errors and overshoot than HCE. However, the cautious controller is also more sluggish than both the dual and HCE modes. It is slow to rise at start-up and is practically inactive during the first 0.3 seconds, which leads to a slower decay of transient

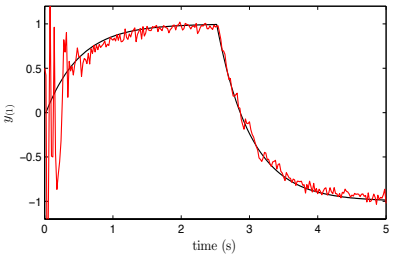

(a) HCE: $y_{(1)}$-red, $y_{d(1)}$-black

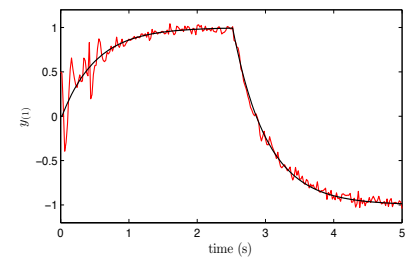

(c) Cautious: $y_{(1)}$-red, $y_{d(1)}$-black

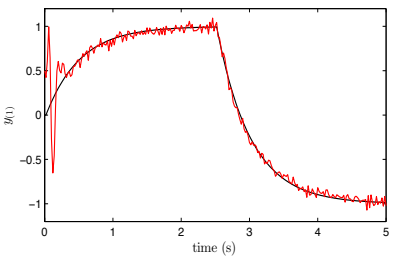

(e) Dual: $y_{(1)}$-red, $y_{d(1)}$-black

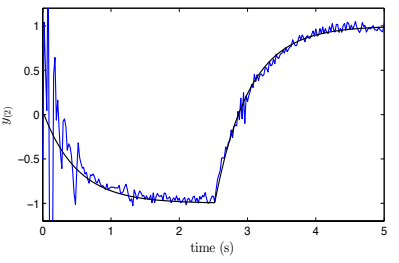

(b) HCE: $y_{(2)}$-blue, $y_{d(2)}$-black

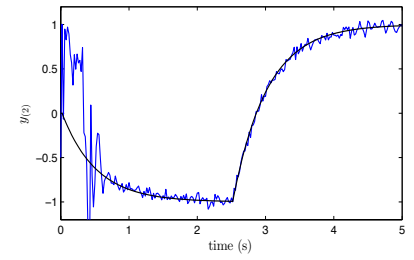
(d) Cautious: $y_{(2)^{-}}$-blue, $y_{d(2)^{-}}$
black

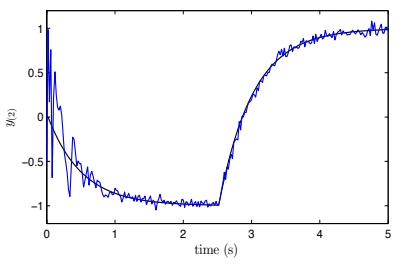

(f) Dual: $y_{(2)}$-blue, $y_{d(2)}$-black
Fig. 2: Single trial results: HCE, cautious and dual 
errors. Thus, as anticipated, the performance of the dual control mode lies midway between the two extremes and qualitatively yields the best transient performance in each of the three schemes, avoiding excessive overshoot and sluggish response as evident in plots (e) and (f). However one should note that in a stochastic system, the information derived form single trial results is only indicative and does not convey reliable performance measures characterizing the system's general average behaviour over time.

In order to address this issue we resort to Monte Carlo analysis where, for each control mode, simulation tests are repeated for 500 trials, each time generating a different noise realization and setting of initial parameters. The reference input vector is unchanged and the tracking performance of each trial is quantified through calculation of a metric $\mathcal{C}$ consisting of the sum of the squared norm of the error between $\boldsymbol{y}_{\boldsymbol{d}}$ and $\boldsymbol{y}$ over a time horizon of 125 sample instants: $\mathcal{C}=\sum_{k=0}^{125}\left\|\boldsymbol{y}_{\boldsymbol{d}}-\boldsymbol{y}_{k}\right\|^{2}$. Operating at a sampling rate of $50 \mathrm{~Hz}$, this corresponds to a time window of $2.5 \mathrm{sec}-$ onds which adequately covers the transient response of the output for this example. Lower values of $\mathcal{C}$ indicate superior control performance. For evaluation purposes, this metric is preferred over cost function (6) because it captures uniquely the tracking capabilities of the controller. Additionally, for comparative analysis, Monte Carlo trials are also performed with the controller's UKF estimator replaced with a standard EKF estimator. This is expected to lead to larger estimation inaccuracy, thereby deteriorating the control performance.

Table I summarizes the outcome of the Monte Carlo tests. It shows the median, mean and variance of $\mathcal{C}$ over 500 trials for each control mode in both the UKF and the EKF-based cases. These three statistical measures are smaller in all control modes of the UKF-based controller than the EKF. This reflects the general superior performance of the UKF estimator, leading to smaller mean and median accumulated cost, as well as variance. Moreover, in both cases, the statistics are lowest for the dual mode case, followed by cautious control and ultimately HCE control. The UKFbased dual mode controller thus gives the overall lowest median, mean and variance measures, reflecting best control performance in a statistically consistent manner.

TABLE I: Statistical measures of the accumulated cost.

\begin{tabular}{|l|c|c|c|}
\hline Controller & Median & Mean & Variance \\
\hline \hline EKF-HCE & 86.13 & 148.6 & $6.3 \times 10^{4}$ \\
\hline EKF-Cautious & 21.65 & 24.24 & 106.3 \\
\hline EKF-Dual & 13.36 & 16.23 & 100.9 \\
\hline UKF-HCE & 74.18 & 106.4 & $1.3 \times 10^{4}$ \\
\hline UKF-Cautious & 16.13 & 18.59 & 82.55 \\
\hline UKF-Dual & 11.76 & 13.62 & 55.67 \\
\hline
\end{tabular}

\section{CONCLUSION}

In this paper we propose a UKF-based MLP neural network controller for dual adaptive control of a class of stochastic MIMO nonlinear systems subject to functional uncertainty. This generalizes the work proposed earlier by the same authors from dual adaptive control of differential mobile robots [9], [16] to a more generic class of MIMO nonlinear systems. This type of adaptive control aims to strike a compromise between the dual control aims of caution and probing, leading to an improved overall response. The performance of the proposed system is evaluated through extensive Monte Carlo analysis of a MIMO nonlinear stochastic example plant. It is shown that the performance of the UKF-based dual adaptive controller is not only consistently superior to HCE and cautious control, but also surpasses the performance of similar controllers which are based on EKF estimation. This reflects the enhanced accuracy of the UKF over the EKF estimator, despite both being local estimation methods. Use of the UKF also avoids the computation of complex Jacobian matrices as part of the design and their evaluation in real-time. These features make the proposed controller very feasible and attractive from a practical implementation point of view. Future work may include an analysis of the convergence and stability properties of the control system. Stability analysis of dual adaptive nonlinear control is an open research challenge for the future [5].

\section{REFERENCES}

[1] A. A. Fel'dbaum, "Dual control theory I-II," Automation and Remote Control, vol. 21, pp. 874-880, 1033-1039, 1960.

[2] —_, "Dual control theory III-IV," Automation and Remote Control, vol. 22, pp. 1-12, 109-121, 1961.

[3] K. J. Åström and B. Wittenmark, Adaptive Control. Mineola, NY, U.S.A: Dover Publications, 2008.

[4] S. G. Fabri and V. Kadirkamanathan, Functional Adaptive Control: An intelligent systems approach. London: Springer-Verlag, 2001.

[5] N. Filatov and H. Unbehauen, Adaptive Dual Control: Theory and applications. Berlin: Springer, 2004.

[6] R. Milito, C. S. Padilla, R. A. Padilla, and D. Cadorin, "An innovations approach to dual control," IEEE Transactions on Automatic Control, vol. AC-27, no. 1, pp. 133-137, February 1982.

[7] C. S. Padilla, J. B. Cruz, and R. A. Padilla, "A simple algorithm for SAFER control," International Journal of Control, vol. 32, no. 6, pp. 1111-1118, 1980.

[8] S. Fabri and V. Kadirkamanathan, "Dual adaptive control of nonlinear stochastic systems using neural networks," Automatica, vol. 34, no. 2, pp. 245-253, February 1998.

[9] M. K. Bugeja and S. G. Fabri, "Dual adaptive control for trajectory tracking of mobile robots," in Proceedings of the IEEE International Conference on Robotics and Automation (ICRA 07), Rome, Italy, April 2007, pp. 2215-2220.

[10] L. Král and M. Šimandl, "Functional adaptive control for multiinput multi-output systems," in Proceedings of the 17th IFAC World Congress, vol. 17. Seoul, South Korea: IFAC, 2008, pp. $11263-$ 11268.

[11] S. J. Julier and J. K. Uhlmann, "Unscented filtering and nonlinear estimation," Proceedings of the IEEE, vol. 92, no. 3, pp. 401-422, 2004.

[12] M. Šimandl and J. Duník, "Sigma point Gaussian sum filter design using square root Unscented filters," in Preprints of the 16th IFAC World Congress, Prague, Czech Republic, July 2005.

[13] S. Haykin, Neural Networks: A comprehensive foundation, 2nd ed. Upper Saddle River, NJ: Prentice-Hall, 1999.

[14] E. A. Wan and R. van der Merwe, "The Unscented Kalman Filter," in Kalman Filtering and Neural Networks, ser. Adaptive and Learning Systems for Signal Processing, Communications and Control, S. Haykin, Ed. John Wiley and Sons, 2001, ch. 7, pp. 221-280.

[15] M. Šmandl, L. Král, and P. Hering, "Neural network based bicriterial dual control of nonlinear systems," in Preprints of the 16th IFAC World Congress, vol. 16, Prague, Czech Republic, July 2005.

[16] M. K. Bugeja, S. G. Fabri, and L. Camilleri, "Dual adaptive control of mobile robots using neural networks," IEEE Transactions on Systems, Man and Cybernetics - Part B, vol. 39, no. 1, pp. 129-141, February 2009. 